\title{
Network Pharmacology Dissection of Multi-scale Mechanisms of Action of Tu-Xian Mixture for the Treatment of Diabetic Cognitive Impairment
}

\section{Bin Yan (D 545020728@qq.com ) \\ Peking Union Medical College Hospital https://orcid.org/0000-0002-2388-1053}

Qing Sun

Peking Union Medical College Hospital

Jianhong Sun

Capital Medical University Affiliated Beijing Friendship Hospital

Guoqing Tian

Peking Union Medical College Hospital

\section{Research}

Keywords: traditional Chinese medicine, network pharmacology, diabetic cognitive medicine, Tu-Xian mixture

Posted Date: December 2nd, 2020

DOI: https://doi.org/10.21203/rs.3.rs-117495/v1

License: (c) (i) This work is licensed under a Creative Commons Attribution 4.0 International License. Read Full License 
5 Running title: Network pharmacology to dissect the mechanisms of

6 traditional Chinese medicine

7

\section{mechanisms of action of Tu-Xian mixture for the treatment} of diabetic cognitive impairment

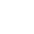

${\text { Bin } \operatorname{Yan}^{1} \text {, Qing Sun }}^{1}$, Jianhong $\operatorname{Sun}^{2}$, Guoqing $\operatorname{Tian}^{1 *}$

1 Department of Traditional Chinese Medicine, Peking Union Medical College

Hospital, Chinese Academy of Medical Sciences, Beijing 100730, China

${ }^{2}$ Beijing Friendship Hospital, Capital Medical University, Beijing 100050, China

Correspondence should be addressed to Guoqing Tian, E-mail: gq-tian@163.com

\section{Word count: 4595}

Figure count: 4

Table count: 9 (including 7 supplementary tables) 


\section{Abstract}

Background Traditional Chinese medicine (TCM) has long been used in the treatment of diabetic cognitive impairment (DCI), and Tu-Xian mixture is one of the prescriptions. This study used a network pharmacology method to predict the active ingredients, potential targets and pathways of Tu-Xian mixture in the treatment of DCI. Methods The potential active ingredients of Tu-xian mixture were obtained by querying databases such as TCMSP, drug targets were screened through Pharmmapper, ETCM, and UniProt databases, and disease target protein groups were collected using TTD, OMIM, and CTD databases. Therapeutic targets were obtained by comparative analysis. Then use WebGestalt to complete GO enrichment analysis and KEGG pathway analysis. Results A total of 51 active ingredients and 619 potential targets were obtained, among which there were 33 key targets for diabetes and 18 key targets for cognitive impairment. After GO analysis and KEGG pathway analysis, steroid hormone biosynthesis and $\gamma$-aminobutyric acid signaling pathway may be the important metabolic pathways for Tu-Xian mixture to perform therapeutic effects.

Conclusions This work promoted the comprehension of Tu-Xian mixture in the treatment of DCI, and further experimental verification of the prediction results was still needed to support its clinical application.

\section{Keywords}

traditional Chinese medicine, network pharmacology, diabetic cognitive medicine, Tu-Xian mixture 


\section{Introduction}

The 9th edition of the IDF Diabetes Atlas released by The International Diabetes Federation (IDF) in 2019 showed that there were about 463 million people with diabetes worldwide, of which about 116 million are in China [1]. Diabetes can cause a variety of chronic complications, and damage to the central nervous system is called diabetic cognitive impairment (DCI). Patients are mainly manifested in decreased ability to learn, memory, and reasoning. Study found that patients with diabetes were more likely to develop cognitive impairment than non-diabetics [2].

Traditional Chinese medicine (TCM) has a complete theoretical system and has unique advantages in the treatment of complex diseases. A systematic review has found that Chinese medicine herbs $(\mathrm{CMH})$ had advantages over conventional western medicine alone in the treatment of DCI [3]. Kidney-reinforcing and blood-activating drugs were commonly used in the treatment of DCI by TCM. In previous work, we have selected some commonly used $\mathrm{CMH}$ based on clinical experience, researched their main extracts, and achieved some results.

Sustained hyperglycemia can cause significant atrophy of hippocampal volume [4, 5], which affects cognitive function in patients with diabetes [6]. Icariin, the main extract of Epimedium brevicornu Maxim (XLP), can reduce hippocampal neuron apoptosis in high glucose environment by upregulating phosphorylation of protein kinase B (Akt) protein [7]. Puerarin, the main extract of Pueraria lobata Ohwi (GG), can inhibit phosphorylation of p38 mitogen-activated protein kinase (MAPK) and c-Jun N-terminal kinase (JNK) to reduce the apoptosis of hippocampal neuron 
cultured in high glucose [8]. Therefore, based on long-term clinical experience and modern drug research [9], we combined a new prescription, namely Tu-Xian mixture. Tu-Xian mixture includes five kinds of $\mathrm{CMH}$, which are Cuscuta australis $\mathrm{R} . \mathrm{Br}$ (TSZ), XLP, Ligustrum lucidum Ait (NZZ), Rhodiola crenulata H. Ohba (HJT), GG.

Network pharmacology is an emerging subject. Through the overall analysis of the "drug-target-gene-disease" of the biological system, the interaction mechanism between the drug and the body is clarified. The systematic and holistic view of this research method is very similar to TCM theory, and network pharmacology has achieved some results in explaining the mechanism of action of TCM [10, 11].

At present, this study analyzed the mechanism of Tu-Xian mixture in the treatment of DCI through network pharmacology. We were expected to find the active ingredients in this prescription that play a therapeutic role and potential targets for treating the disease. The obtained results, we hope, may not only promote the comprehension of Tu-Xian mixture in the treatment of DCI, but also promote TCM as a more important alternative therapy in the treatment of complex diseases.

\section{Materials and methods}

\subsection{Construction of database and active ingredients screening}

The ingredients of all herbs in Tu-Xian mixture came from related literature and databases, including Traditional Chinese Medicine Systems Pharmacology Database and Analysis Platform (TCMSP, http://tcmspw.com/tcmsp.php, accessed August 2019)
[12], Traditiona
Chinese
Medicines Integrated
Database
( TCMID, 
http://119.3.41.228:8000/tcmid/, accessed August 2019) [13], a Bioinformatics Analysis Tool for Molecular mechANism of Traditional Chinese Medicine (BATMAN-TCM, http://bionet.ncpsb.org/batman-tcm/index.php/Home/Index/index, accessed August 2019) [14], and Chemistry Database of Chinese Academy of Sciences (www.organchem.csdb.cn, accessed August 2019). Then the ingredients were screened according to oral bioavailability (OB) and drug-likeness (DL). Oral bioavailability is one of the most basic pharmacokinetic properties of oral drugs, which refers to the relative absorption of the drug into the blood circulation after oral administration [15]. When TCM decoction is taken orally, there may be hundreds of ingredients entering the body simultaneously, and only a part of which has a higher OB can play a therapeutic role. Drug-likeness is a qualitative analysis method to assess whether a molecule is suitable as a drug by evaluating the chemical properties [16]. According to the recommendation of the TCMSP database, ingredients that meet $\mathrm{OB} \geq 30 \%$ and $\mathrm{DL} \geq 0.18$ were reserved for further analysis.

\subsection{Drug targets prediction}

Potential drug targets consisted of two parts. Firstly, retrieve the above potential ingredients through Pubchem server (https://pubchem.ncbi.nlm.nih.gov/, accessed August 2019), download their structural files, and upload them to the Pharmmapper server (http://www.lilab-ecust.cn/pharmmapper/, accessed August 2019) [17]. The Pharmmapper server can perform target prediction through reverse pharmacophore matching. After obtaining the result, retain the targets with Norm Fit $\geqslant 0.7$, and 
111

112

113

convert them from Uniprot code to Gene Symbol standard code. Secondly, retrieve the above potential ingredients through The Encyclopedia of Traditional Chinese Medicine (ETCM, http://www.nrc.ac.cn:9090/ETCM/index.php/Home/Index/, accessed August 2019), get the predicted targets directly provided by this server. The two parts were combined and analyzed to obtain all potential drug targets.

\subsection{Known therapeutic targets of DCI}

At present, the exact pathogenesis of DCI is unclear, but numerous studies have found that there were many of the same pathological changes between DCI and Alzheimer's disease (AD)[18-20]. Therefore, in the absence of DCI targets, we collected the targets of diabetes and $\mathrm{AD}$ as the disease targets of DCI. Then using "Diabetes" and "Alzheimer's Disease" as keywords, they were searched and screened in Therapeutic Target Database (TTD, http://bidd.nus.edu.sg/group/cjttd/, accessed September 2019)[21], Online Mendelian Inheritance in Man (OMIM, https://omim.org/, accessed September 2019)[22], and Comparative Toxicogenomics Database (CTD, http://ctdbase.org/, accessed September 2019)[23]. Integrate the target of diabetes and AD separately, and compare and analyze with the potential drug targets of the previous step. Thus, we obtained the relevant targets of DCI that Tu-Xian Mixture may act on.

\subsection{Protein-protein interaction and network construction} Import the therapeutic targets into STRING (https://string-db.org/, accessed 
133

134

October 2019)[24] and download the protein-protein interaction (PPI) file. Introduce the PPI file into the visual tool Cytoscape (version 3.7.1, Boston, MA, USA) to analyze the topological parameters of the PPI network. Node degree, a very important topological parameter, is the number of connections between nodes, which can reflect the importance of a node in the network. To improve the accuracy of the results, those with a node degree above the median were key targets.

\subsection{GO enrichment analysis and KEGG pathway analysis}

Complete the GO enrichment analysis and KEGG pathway analysis of the selected key targets through the WebGestalt (http://www.webgestalt.org/, accessed October 2019) [25] online server. The GO term in biological processes indicated the function of genes, and the KEGG term symbolized a specific function of a gene in a metabolic pathway. Those GO and KEGG terms with $p$-value $\leq 0.05$ and false discovery rate (FDR) $\leq 0.05$ were significant, which represents the function of key targets and the metabolic pathways involved.

\subsection{Workflow}

The specific workflow is shown in Figure 1. Firstly, the active ingredients of Tu-Xian mixture were screened by searching the databases, and they needed to satisfy the screening requirements, $\mathrm{OB} \geq 30 \%$ and $\mathrm{DL} \geq 0.18$. Secondly, the databases were also used to screen potential therapeutic targets of active ingredients and disease targets of DCI, respectively. Subsequently, construct a network model and complete 
the enrichment GO analysis and KEGG pathway analysis.
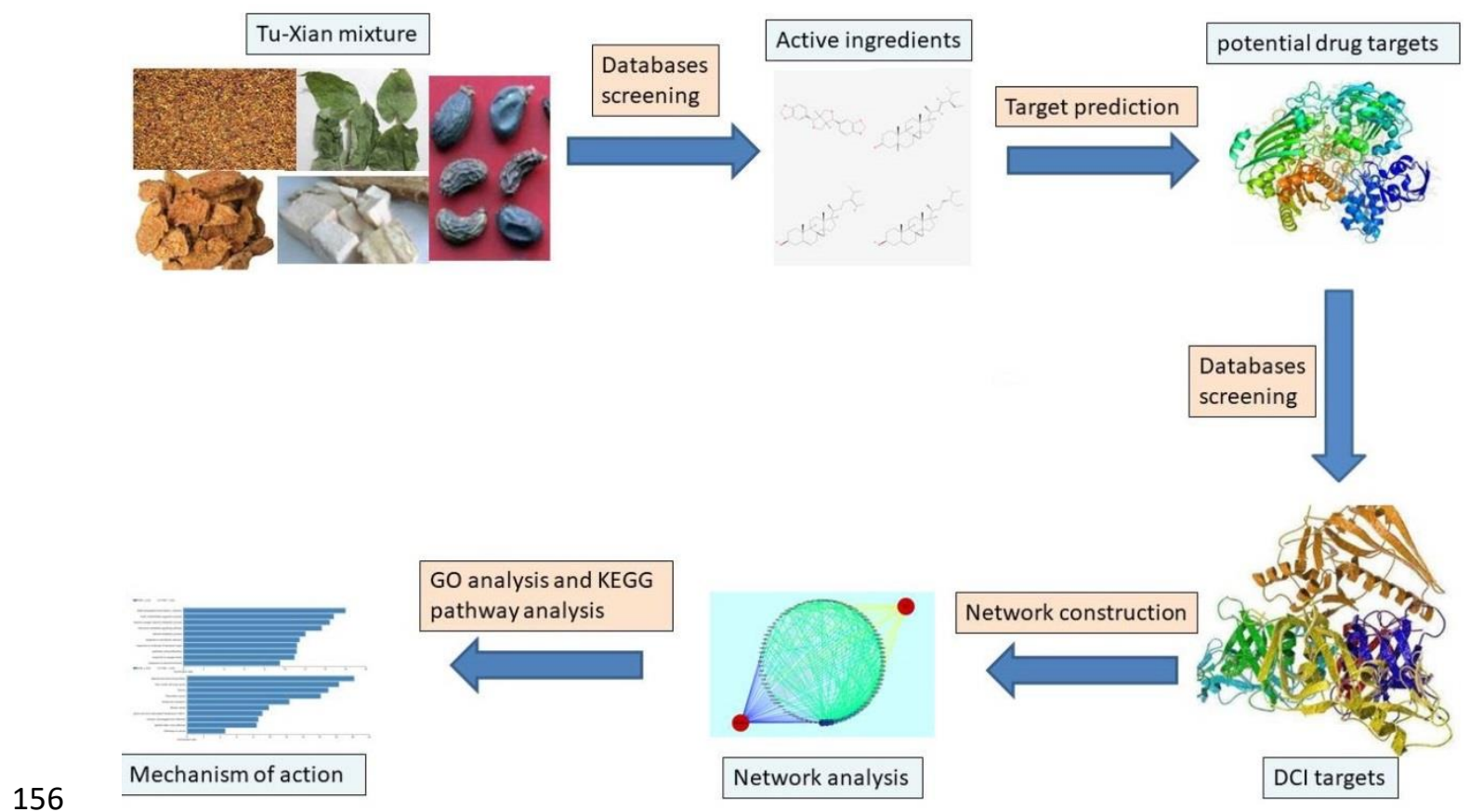

Figure 1 Workflow of the approach

3. Results

161 displays part of them as examples. 


\begin{tabular}{ccccc}
\hline No. & Latin name & Chinese pinyin & Abbreviation & Ingredients \\
\hline 1 & Cuscuta australis $\mathrm{R} . \mathrm{Br}$ & Tu si zi & TSZ & 35 \\
2 & Epimedium brevicornu Maxim & Xian ling pi & XLP & 139 \\
3 & Ligustrum lucidum Ait & Nv zhen zi & NZZ & 153 \\
4 & Rhodiola crenulata H. Ohba & Hong jing tian & HJT & 26 \\
5 & Pueraria lobata Ohwi & Ge gen & GG & 54 \\
\hline
\end{tabular}

\begin{tabular}{|c|c|c|c|c|}
\hline Molecule ID & Ingredient name & $\mathrm{OB}(\%)$ & DL & Structure \\
\hline MOL001558 & sesamin & 56.55 & 0.83 & \\
\hline MOL000184 & NSC63551 & 39.25 & 0.76 & \\
\hline MOL005440 & Isofucosterol & 43.78 & 0.76 & \\
\hline
\end{tabular}




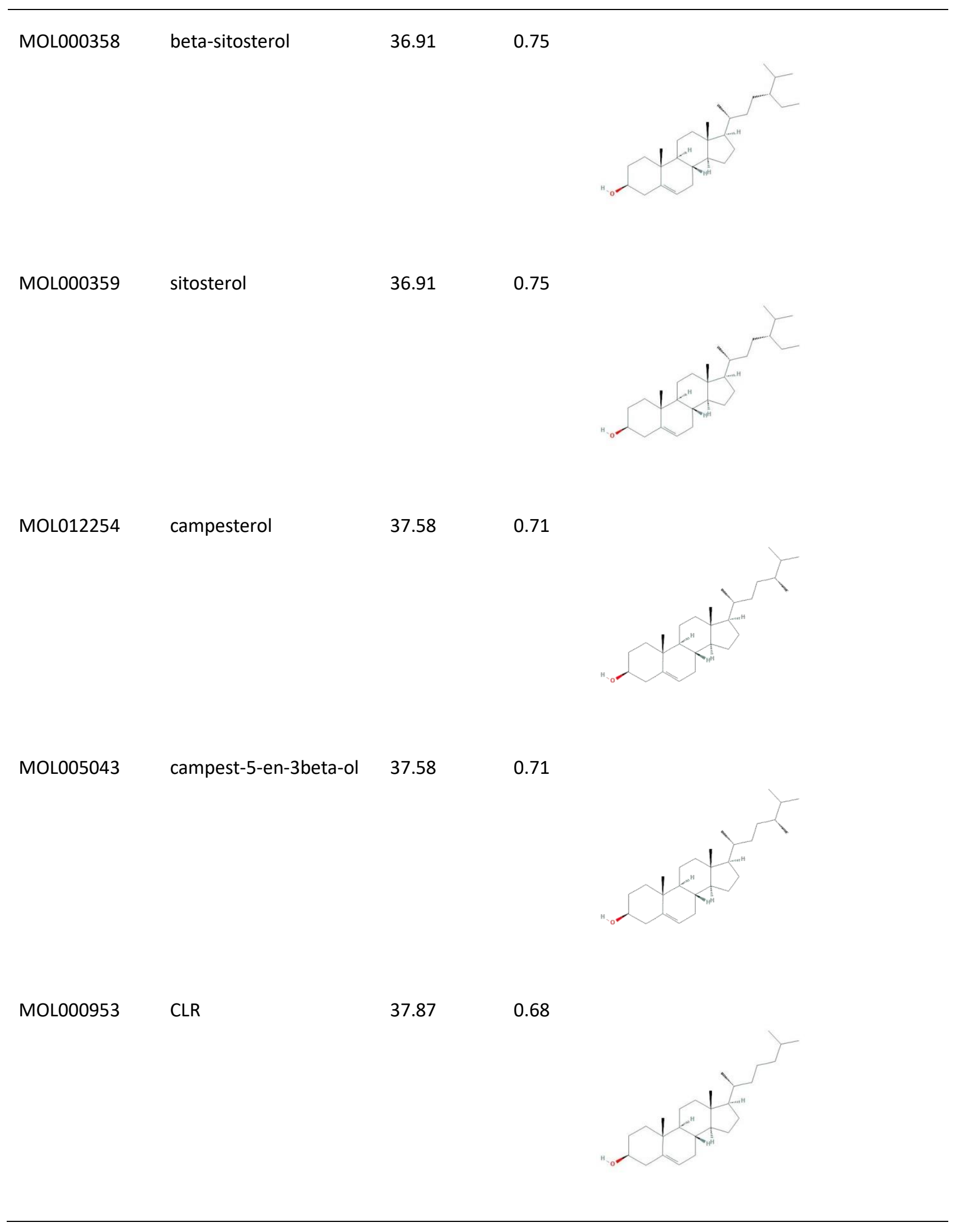




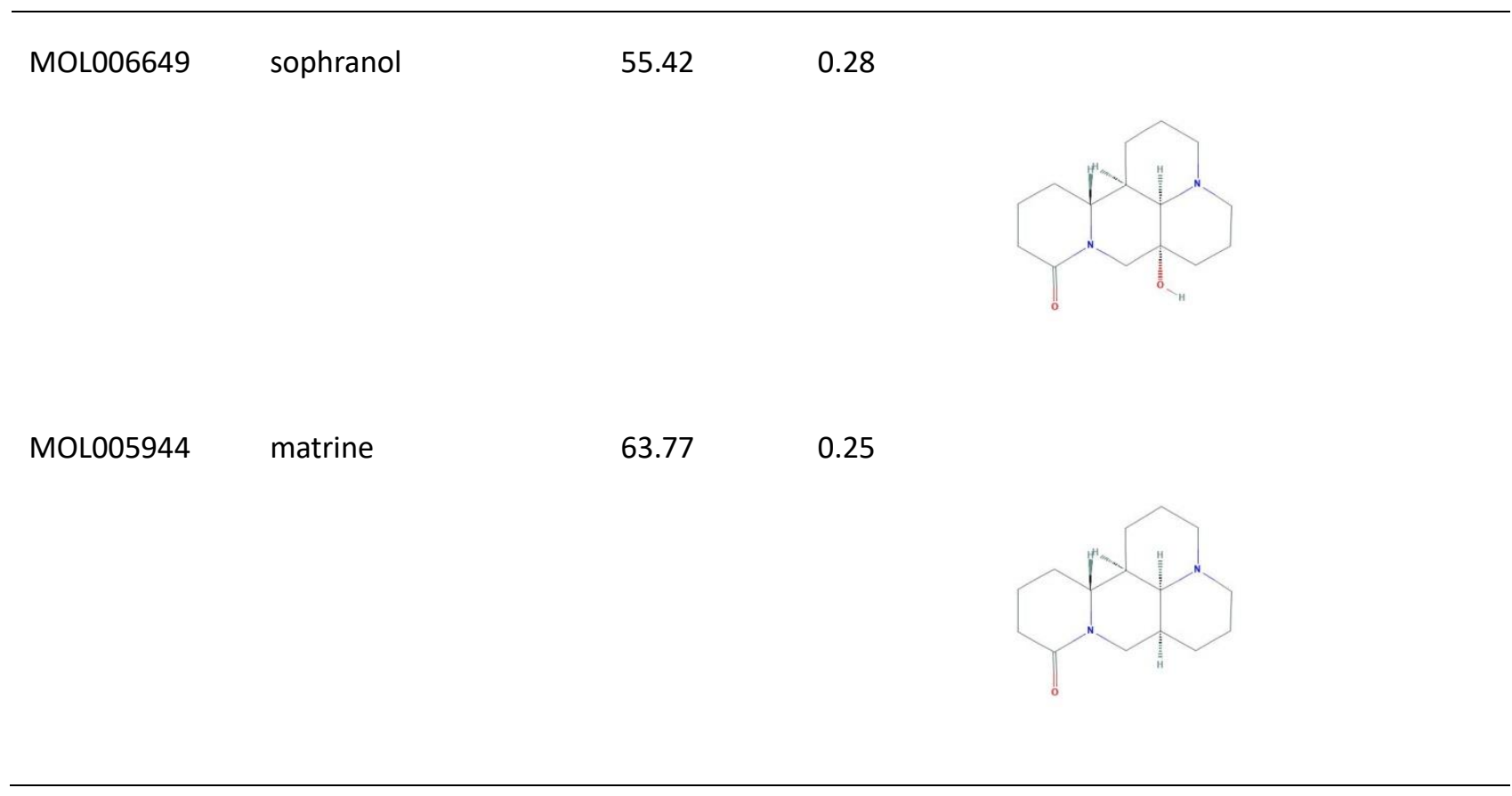

173 Molecule ID was provided by TCMSP; OB, oral bioavailability; DL, drug-likeness.

174

175 3.2. Potential therapeutic targets.

176 A total of 619 potential drug targets of the above 51 active ingredients were

177 obtained through Pharmmapper and the ETCM databases. Detailed information about

178 these targets was provided in Supplementary Table S2. In total, 1158 diabetes targets 179 and 437 AD targets were obtained from TTD, OMIM and CTD databases (Table S3).

180 After a comparative analysis of potential drug targets and disease targets, we 181 demonstrated that Tu-Xian mixture might affect 64 diabetes-related targets and 32 182 AD-related targets (Table S4).

183

$184 \quad 3.3$. Network and visual analysis

185 Upload the above potential therapeutic targets of diabetes and AD to the STRING 186 database, and the obtained PPI relationship was introduced into Cytoscape for visual 

(Table S5).

analysis (Figure 2). The nodes with degree above the median were selected as the key targets, and 33 key targets for diabetes and 18 key targets for $\mathrm{AD}$ were obtained

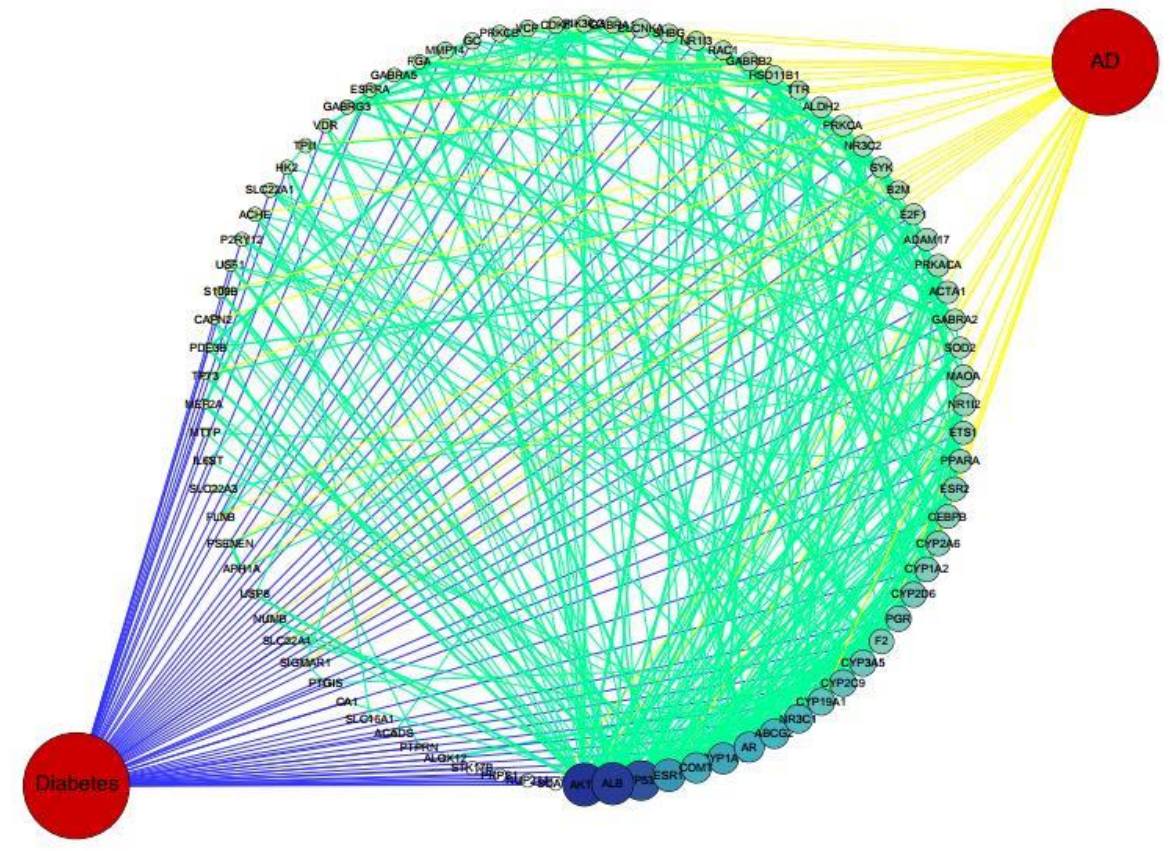

190

Figure 2 The PPI between potential therapeutic targets of diabetes and AD

AD, Alzheimer's disease. Circles represent diseases or targets. The circle size of targets is proportional to its

degree. The green, blue, and yellow lines indicate the target-to-target, diabetes-to-target, and AD-to-target links, respectively.

\subsection{GO enrichment analysis and KEGG pathway analysis}

To explore the biological processes and metabolic pathways involved in the above key targets, we further conducted GO analysis and KEGG pathway analysis. Our results were presented in two parts, diabetes-key-target and AD-key-target. 


\subsubsection{Diabetes-key-target}

The results of GO enrichment analysis suggested that the biological processes of the diabetes key targets mainly included DNA-templated transcription, initiation, hormone-mediated signaling pathway, steroid metabolic process, and responses to steroid hormone. And KEGG pathway analysis suggested that the signal pathways involved in these key genes mainly included three functional modules, namely endocrine metabolism (steroid hormone biosynthesis), cancer, and infection (Figure 3, with their p-value and FDR shown in Supplementary Table S6). Among them, the biosynthesis of steroid hormones has a high enrichment rate in both GO analysis and KEGG pathway analysis, which indicated that $\mathrm{Tu}$-Xian mixture may play a role in treating diabetes by regulating steroid hormones.

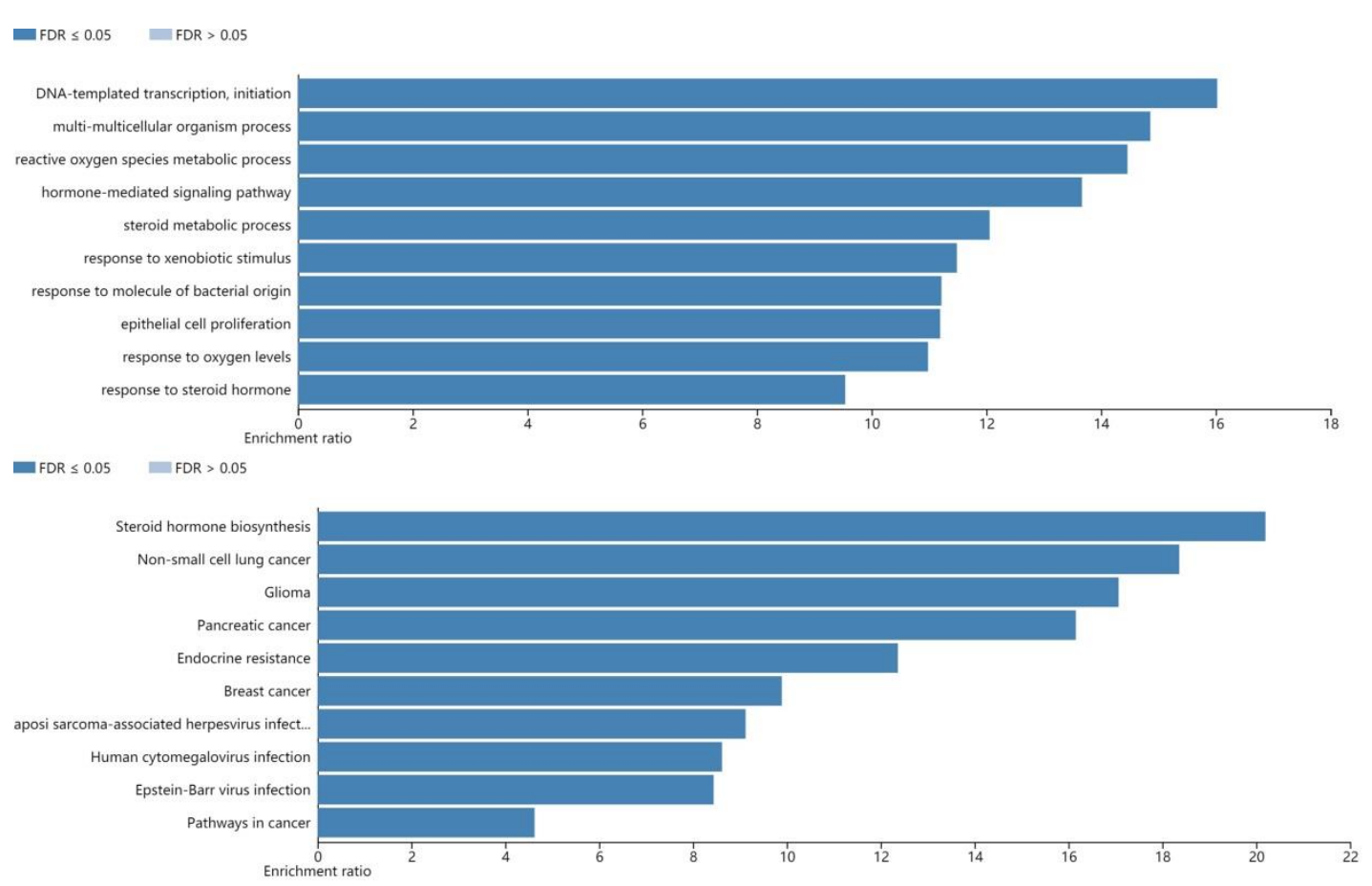

Figure 3 GO enrichment analysis and KEGG pathway analysis of diabetes key targets

The $\mathrm{y}$-axis represents the type of biological process or signaling pathway, and the $\mathrm{x}$-axis 
represents the enrichment ratio of these items ( $p$-value $\leq 0.05$ and FDR $\leq 0.05$ ).

\subsubsection{AD-key-target}

Similar steps as above, from the results of GO enrichment analysis, it could be seen that key genes were mainly involved in the $\gamma$-aminobutyric acid signaling pathway, synaptic transmission, GABAergic, steroid metabolic process, regulation of

222 neurotransmitter levels, and neuronal death. And KEGG pathway analysis results 223 suggested that the enriched signaling pathways mainly included neural signaling 224 (GABAergic synapse, retrograde endocannabinoid signaling, neuroactive 225 ligand-receptor interaction), drug addiction and endocrine metabolism (Figure 4, with 226 their p-value and FDR shown in Supplementary Table S7). We found that the $227 \gamma$-aminobutyric acid signaling pathway has a higher enrichment rate in both GO analysis and KEGG pathway analysis. Therefore, Tu-Xian mixture might improve cognitive function mainly by regulating the $\gamma$-aminobutyric acid signaling pathway. 


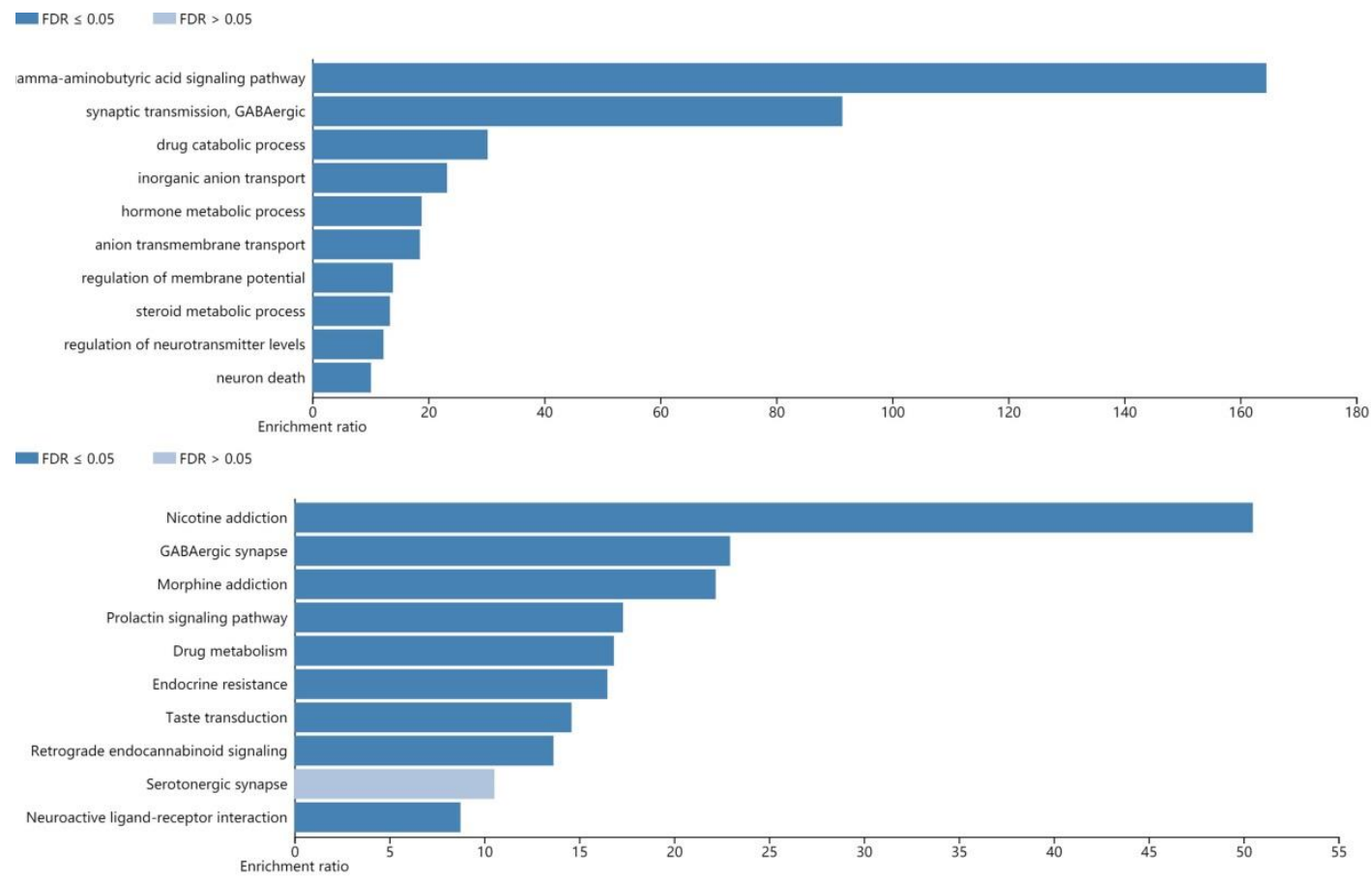

Figure $4 \mathrm{GO}$ enrichment analysis and KEGG pathway analysis of AD key targets

The $\mathrm{y}$-axis represents the type of biological process or signaling pathway, and the $\mathrm{x}$-axis represents the enrichment ratio of these items ( $p$-value $\leq 0.05$ and FDR $\leq 0.05$ ).

\section{Discussion}

Previous studies have found that the five herbs or their ingredients in Tu-Xian mixture have certain effects in the treatment of diabetes and cognitive impairment.

239 Cuscuta australis R.Br (TSZ) can improve memory function in rats with cognitive 240 impairment induced by scopolamine by restoring cholinergic function, reducing oxidative stress and neuroinflammation [26]. Ligustrum lucidum Ait (NZZ) can reduce fasting blood glucose in diabetic rats and protect the islet $\beta$ cells[27]. Multiple

243 extracts of XLP can improve diabetes complications [28, 29], and icariside II can 244 reduce cognitive deficits in rats with cognitive impairment induced by neurotoxins 245 [30]. Puerarin has hypoglycemic effect and can improve insulin resistance [31] and 
diabetes complications [32] in diabetic mice, and it can reduce cognitive impairment

247 in precursor protein/presenilin-1 double transgenic model mice [33]. Salidroside has

248 been found to reduce fasting blood glucose and blood lipid levels in diabetic rats, and 249 to prevent cognitive impairment caused by diabetes by regulating the 250 Rho/Rho-associated kinase (ROCK)/sirtuin 1 (SIRT1)/nuclear factor (NF)- $\mathrm{BB}$ 251 pathway[9].

252 By searching multiple databases, the number of targets that Tu-Xian mixture can 253 affect diabetes and $\mathrm{AD}$ was 33 and 18, respectively. After GO analysis and KEGG 254 pathway analysis, the results suggested that Tu-Xian mixture mainly works through 255 three functional pathways in the treatment of diabetes: steroid hormone biosynthesis, 256 cancer and infection. And the key functional pathway is steroid hormone biosynthesis.

257 Similarly, when treating cognitive impairment, there were three functional pathways:

258 neural signal transduction, drug addiction, and endocrine metabolism. Obviously, 259 neural signal transduction played a leading role.

260 Steroid hormone biosynthesis is closely related to the occurrence and 261 development of diabetes. A health survey report showed that low testosterone and 262 high estradiol levels were associated with obesity in men and increased insulin 263 resistance and risk of diabetes [34]. Prospective studies found low levels of 264 testosterone as risk factor for diabetes in men [35, 36], and men with low levels of 265 testosterone had 2.3 times more diabetes than normal men[37]. Therefore, low levels 266 of testosterone were closely related to the occurrence and development of disease in 267 male diabetic patients. The possible mechanism is that testosterone can inhibit 
lipoprotein lipase activity, increase the breakdown and utilization of fat and after testosterone levels decrease, fat deposition increases and insulin sensitivity decreases [38]. And low testosterone levels could inhibit glycogen synthesis leading to muscle-level insulin resistance [39]. For women, the imbalance of steroid hormones was also closely related to the occurrence and development of diabetes [40, 41]. Among them, sex hormone binding globulin was negatively correlated with the onset of diabetes, and estradiol is positively correlated with the onset of diabetes. Animal experiments indicated that increased muscle steroid hormones can increase insulin sensitivity in type 2 diabetic rats [42], which may be achieved by activating the Akt / PKC- $\zeta / \lambda$-GLUT-4 signal pathway [43]. Therefore, the application of adjuvant hormone therapy for the treatment of diabetes has a broad prospect. This may also be an important way for Tu-Xian mixture to treat diabetes.

The relationship between diabetes and cancer is extremely close. A meta-analysis of a prospective cohort study involving 900,000 participants showed that prediabetes was closely related to the occurrence of cancer [44], and the common molecular mechanism might be the bridge between them [45]. Diabetes is also closely related to infection. Studies showed that people with diabetes had a significantly higher risk of infection than ordinary people [46]. Therefore, Tu-Xian mixture may achieve the therapeutic effect on diabetes and its complications by regulating cancer and infection related pathways. $\gamma$-aminobutyric acid is an important neurotransmitter with various physiological functions. Its neurons mainly exist in the cerebral cortex, hippocampus, and striatum. 
$\gamma$-aminobutyric acid can inhibit the activity of excitatory neurons such as the cortex

291 and hippocampus, and thus maintain normal neural network activity in the brain.

Brain excitement suppresses imbalance induced by $\gamma$-aminobutyric acid system dysfunction is an important mechanism for cognitive impairment [47, 48]. The levels of $\gamma$-aminobutyric acid in the brain of patients with opioid addiction were significantly reduced, which was positively related to cognitive impairment [49]. Alpha-asarone, the major ingredient isolated from the Chinese medicinal herb Acorus tatarinowii Schott, can improve the cognitive function of $\mathrm{AD}$ rats by enhancing the $\gamma$-aminobutyric acid system, inhibiting the overexcitation of hippocampal neurons, and reducing the deposition of amyloid $\beta$-protein $(\mathrm{A} \beta)[50]$. Therefore, $\gamma$-aminobutyric acid is involved in the development of cognitive impairment, and its specific mechanism in DCI needs further study. This research lays the theoretical foundation for the next experimental verification.

\section{Conclusion}

In this study, a network pharmacology research method was used to reveal the chemical basis of Tu-Xian mixture and explore the mechanism of Tu-Xian Mixture in the treatment of DCI. Total 51 active ingredients were selected from Tu-Xian mixture, and 619 potential drug targets were identified. Among them, there were 33 targets for diabetes treatment and 18 targets for cognitive impairment treatment, which play a therapeutic role by regulating steroid hormone biosynthesis and $\gamma$-aminobutyric acid signaling pathway. This work provided a theoretical basis for the study of Tu-Xian 
mixture on the pharmacological mechanism of DCI. Further experimental verification of the prediction results is still needed to support its clinical application.

\section{List of abbreviations}

AD: Alzheimer's disease; BATMAN-TCM: a Bioinformatics Analysis Tool for Molecular mechANism of Traditional Chinese Medicine; $\mathrm{CMH}$ : Chinese medicine herbs; CTD: Comparative Toxicogenomics Database; DCI: diabetic cognitive impairment; DL: drug-likeness; ETCM: Encyclopedia of Traditional Chinese Medicine; GG: Ge gen; HJT: Hong jing tian; IDF: International Diabetes Federation; NZZ: Nv zhen zi; OB: oral bioavailability; OMIM: Online Mendelian Inheritance in Man; TCM: Traditional Chinese medicine; TCMID: Traditional Chinese Medicines Integrated Database; TCMSP: Traditional Chinese Medicine Systems Pharmacology Database and Analysis Platform; TSZ: Tu si zi; TTD: Therapeutic Target Database; XLP: Xian ling pi.

\section{Ethics approval and consent to participate} Not applicable.

\section{Consent to publish} Not applicable.

\section{Availability of data and material}



corresponding author on reasonable request.

\section{Competing interests} The authors declared no conflict of interests.

All authors reviewed the manuscript. the email.

[2] Cukierman T, Gerstein HC, Williamson JD. Cognitive decline and dementia in diabetessystematic overview of prospective observational studies. Diabetologia 2005;48(12):2460. Impairment: A Systematic Review and Meta-Analysis. Evid-Based Compl Alt 2018;2018:1-10. 


\section{doi:10.1155/2018/7541406}

[4] Geijselaers S, Sep S, Stehouwer C, Biessels GJ. Glucose regulation, cognition, and brain MRI in type 2 diabetes: a systematic review. Lancet Diabetes Endocrinol 2015;3(1):75-89. doi:10.1016/S2213-8587(14)70148-2

[5] Moran C, Phan TG, Chen J, Blizzard L, Beare R, Venn A, et al. Brain atrophy in type 2 diabetes: regional distribution and influence on cognition. Diabetes Care 2013;36(12):4036-42. doi:10.2337/dc13-0143

[6] Gold SM, Dziobek I, Sweat V, Tirsi A, Rogers K, Bruehl H, et al. Hippocampal damage and memory impairments as possible early brain complications of type 2 diabetes. Diabetologia 2007;50(4):711-9. doi:10.1007/s00125-007-0602-7

[7] Yan B, Wang J, Zhang H, Tian G, Liu Y. Effect of icariin on apoptosis in hippocampal neurons cultured in high glucose. J Tradit Chin Med 2018;38(04):556-61.

[8] Xu X, Wang J, Zhang H, Tian G, Liu Y. Puerarin reduces apoptosis in rat hippocampal neurons culturea in high glucose medium by modulating the p38 mitogen activated protein kinase and c-Jun N-terminal kinase signaling pathways. J Tradit Chin Med 2016;36(1):78-84.

[9] Hao X, Yuan J, Dong H. Salidroside prevents diabetesinduced cognitive impairment via regulating the Rho pathway. Mol Med Rep 2019;19(1):678-84. doi:10.3892/mmr.2018.9621

[10] Gao K, Yang R, Zhang J, Wang Z, Jia C, Zhang F, et al. Effects of Qijian mixture on type 2 diabetes assessed by metabonomics, gut microbiota and network pharmacology. Pharmacol Res 2018;130:93-109. doi:10.1016/j.phrs.2018.01.011

[11] Zhao M, Chen Y, Wang C, Xiao W, Chen S, Zhang S, et al. Systems Pharmacology Dissection of Multi-Scale Mechanisms of Action of Huo-Xiang-Zheng-Qi Formula for the Treatment of Gastrointestinal Diseases. Front Pharmacol 2018;9:1448. doi:10.3389/fphar.2018.01448

[12] Ru J, Li P, Wang J, Zhou W, Li B, Huang C, et al. TCMSP: a database of systems pharmacology for drug discovery from herbal medicines. J Cheminform 2014;6:13. doi:10.1186/1758-2946-6-13

[13] Huang L, Xie D, Yu Y, Liu H, Shi Y, Shi T, et al. TCMID 2.0: a comprehensive resource for TCM. Nucleic Acids Res 2017;46. doi:10.1093/nar/gkx1028

[14] Liu Z, Guo F, Wang Y, Li C, Zhang X, Li H, et al. BATMAN-TCM: a Bioinformatics Analysis Tool for Molecular mechANism of Traditional Chinese Medicine. Sci Rep 2016;6:21146. doi:10.1038/srep21146

[15] Xu X, Zhang W, Huang C, Li Y, Yu H, Wang Y, et al. A novel chemometric method for the prediction of human oral bioavailability. Int J Mol Sci 2012;13(6):6964-82. doi:10.3390/ijms13066964

[16] Tao W, Xu X, Wang X, Li B, Wang Y, Li Y, et al. Network pharmacology-based prediction of the active ingredients and potential targets of Chinese herbal Radix Curcumae formula for application to cardiovascular disease. J Ethnopharmacol 2013;145(1):1-10. doi:10.1016/j.jep.2012.09.051

[17] Wang X, Shen Y, Wang S, Li S, Zhang W, Liu X, et al. PharmMapper 2017 update: a web server for potential drug target identification with a comprehensive target pharmacophore database. Nucleic Acids Res 2017;45(W1):W356-60. doi:10.1093/nar/gkx374

[18] Kimura N. Diabetes Mellitus Induces Alzheimer's Disease Pathology: Histopathological Evidence from Animal Models. Int J Mol Sci 2016;17(4):503. doi:10.3390/ijms17040503

[19] Talfournier J, Bitu J, Paquet C, Gobron C, Guillausseau PJ, Hugon J, et al. Relationship between blood pressure, cognitive function and education level in elderly patients with diabetes: a preliminary study. Diabetes Metab 2013;39(5):418-23. doi:10.1016/j.diabet.2013.02.008 
[20] de la Monte SM. Type 3 diabetes is sporadic Alzheimers disease: mini-review. Eur Neuropsychopharmacol 2014;24(12):1954-60. doi:10.1016/j.euroneuro.2014.06.008

[21] Li YH, Yu CY, Li XX, Zhang P, Tang J, Yang Q, et al. Therapeutic target database update 2018: enriched resource for facilitating bench-to-clinic research of targeted therapeutics. Nucleic Acids Res 2018;46(D1):D1121-7. doi:10.1093/nar/gkx1076

[22] Amberger JS, Hamosh A. Searching Online Mendelian Inheritance in Man (OMIM): A Knowledgebase of Human Genes and Genetic Phenotypes. Curr Protoc Bioinformatics 2017;58:1-2. doi:10.1002/cpbi.27

[23] Davis AP, Grondin CJ, Johnson RJ, Sciaky D, McMorran R, Wiegers J, et al. The Comparative Toxicogenomics Database: update 2019. Nucleic Acids Res 2019;47(D1):D948-54. doi:10.1093/nar/gky868

[24] Szklarczyk D, Gable AL, Lyon D, Junge A, Wyder S, Huerta-Cepas J, et al. STRING v11: protein-protein association networks with increased coverage, supporting functional discovery in genome-wide experimental datasets. Nucleic Acids Res 2019;47(D1):D607-13. doi:10.1093/nar/gky1131

[25] Liao Y, Wang J, Jaehnig EJ, Shi Z, Zhang B. WebGestalt 2019: gene set analysis toolkit with revamped UIs and APIs. Nucleic Acids Res 2019;47(W1):W199-205. doi:10.1093/nar/gkz401

[26] Lin MK, Lee MS, Huang HC, Cheng TJ, Cheng YD, Wu CR. Cuscuta chinensis and C. campestris Attenuate Scopolamine-Induced Memory Deficit and Oxidative Damage in Mice. Molecules 2018;23(12). doi:10.3390/molecules23123060

[27] Lee SI, Oh SH, Park KY, Park BH, Kim JS, Kim SD. Antihyperglycemic effects of fruits of privet (Ligustrum obtusifolium) in streptozotocin-induced diabetic rats fed a high fat diet. J Med Food 2009;12(1):109-17. doi:10.1089/jmf.2007.0000

[28] Nakashima K, Miyashita H, Yoshimitsu H, Fujiwara Y, Nagai R, Ikeda T. Two new prenylflavonoids from Epimedii Herba and their inhibitory effects on advanced glycation end-products. J Nat Med 2016;70(2):290-5. doi:10.1007/s11418-015-0962-0

[29] Zhou F, Xin H, Liu T, Li GY, Gao ZZ, Liu J, et al. Effects of icariside II on improving erectile function in rats with streptozotocin-induced diabetes. J Androl 2012;33(5):832-44. doi:10.2164/jandrol.111.015172

[30] He L, Deng Y, Gao J, Zeng L, Gong Q. Icariside II ameliorates ibotenic acid-induced cognitive impairment and apoptotic response via modulation of MAPK pathway in rats. Phytomedicine 2018;41:74-81. doi:10.1016/j.phymed.2018.01.025

[31] Chen X, Qian L, Wang B, Zhang Z, Liu H, Zhang Y, et al. Synergistic Hypoglycemic Effects of Pumpkin Polysaccharides and Puerarin on Type II Diabetes Mellitus Mice. Molecules 2019;24(5). doi:10.3390/molecules24050955

[32] Pan X, Wang J, Pu Y, Yao J, Wang H. Effect of Puerarin on Expression of ICAM-1 and TNF-alpha in Kidneys of Diabetic Rats. Med Sci Monit 2015;21:2134-40. doi:10.12659/MSM.893714

[33] Zhou Y, Xie N, Li L, Zou Y, Zhang X, Dong M. Puerarin alleviates cognitive impairment and oxidative stress in APP/PS1 transgenic mice. Int J Neuropsychopharmacol 2014;17(4):635-44. doi:10.1017/S146114571300148X

[34] Li J, Lai H, Chen S, Zhu H, Lai S. Interaction of sex steroid hormones and obesity on insulin resistance and type 2 diabetes in men: the Third National Health and Nutrition Examination Survey. J Diabetes Complications 2017;31(2):318-27. doi:10.1016/j.jdiacomp.2016.10.022 
[35] Joyce KE, Biggs ML, Djoussé L, Ix JH, Kizer JR, Siscovick DS, et al. Testosterone, Dihydrotestosterone, Sex Hormone Binding Globulin and Incident Diabetes among Older Men: the Cardiovascular Health Study. The Journal of Clinical Endocrinology \& Metabolism 2016:2016-623. doi:10.1210/jc.2016-2623

[36] Salminen M, Vahlberg T, Räihä I, Niskanen L, Kivelä S, Irjala K. Sex hormones and the risk of type 2 diabetes mellitus: A 9-year follow up among elderly men in Finland. Geriatr Gerontol Int 2015;15(5):559-64. doi:10.1111/ggi.12312

[37] Laaksonen DE, Niskanen L, Punnonen K, Nyyssonen K, Tuomainen TP, Valkonen VP, et al. Testosterone and sex hormone-binding globulin predict the metabolic syndrome and diabetes in middle-aged men. Diabetes Care 2004;27(5):1036-41. doi:10.2337/diacare.27.5.1036

[38] Schianca GP, Fra GP, Brustia F, Bellan M, Pirovano A, Gualerzi A, et al. Testosterone Plasma Concentration is Associated with Insulin Resistance in Male Hypertensive Patients. Exp Clin Endocrinol Diabetes 2017;125(3):171-5. doi:10.1055/s-0042-121492

[39] Basaria S, Muller DC, Carducci MA, Egan J, Dobs AS. Hyperglycemia and insulin resistance in men with prostate carcinoma who receive androgen-deprivation therapy. Cancer-Am Cancer Soc 2006;106(3):581-8. doi:10.1002/cncr.21642

[40] Kim NN. Sex steroid hormones in diabetes-induced sexual dysfunction: focus on the female gender. J Sex Med 2009;6 Suppl 3:239-46. doi:10.1111/j.1743-6109.2008.01182.x

[41] Muka T, Nano J, Jaspers L, Meun C, Bramer WM, Hofman A, et al. Associations of Steroid Sex Hormones and Sex Hormone-Binding Globulin With the Risk of Type 2 Diabetes in Women: A Population-Based Cohort Study and Meta-analysis. Diabetes 2017;66(3):577-86. doi:10.2337/db16-0473

[42] Sato K, Fujita S, Iemitsu M. Dioscorea esculenta-induced increase in muscle sex steroid hormones is associated with enhanced insulin sensitivity in a type 2 diabetes rat model. Faseb $\mathbf{J}$ 2017;31(2):793-801. doi:10.1096/fj.201600874R

[43] Sato K, Fujita S, Iemitsu M. Acute administration of diosgenin or dioscorea improves hyperglycemia with increases muscular steroidogenesis in STZ-induced type 1 diabetic rats. J Steroid Biochem Mol Biol 2014;143:152-9. doi:10.1016/j.jsbmb.2014.02.020

[44] Huang Y, Cai X, Qiu M, Chen P, Tang H, Hu Y, et al. Prediabetes and the risk of cancer: a meta-analysis. Diabetologia 2014;57(11):2261-9. doi:10.1007/s00125-014-3361-2

[45] Hua F, Yu JJ, Hu ZW. Diabetes and cancer, common threads and missing links. Cancer Lett 2016;374(1):54-61. doi:10.1016/j.canlet.2016.02.006

[46] Shah BR, Hux JE. Quantifying the risk of infectious diseases for people with diabetes. Diabetes Care 2003;26(2):510-3. doi:10.2337/diacare.26.2.510

[47] Marenco S, Meyer C, van der Veen JW, Zhang Y, Kelly R, Shen J, et al. Role of gamma-amino-butyric acid in the dorsal anterior cingulate in age-associated changes in cognition. Neuropsychopharmacol 2018;43(11):2285-91. doi:10.1038/s41386-018-0134-5

[48] Oeltzschner G, Wijtenburg SA, Mikkelsen M, Edden R, Barker PB, Joo JH, et al. Neurometabolites and associations with cognitive deficits in mild cognitive impairment: a magnetic resonance spectroscopy study at 7 Tesla. Neurobiol Aging 2019;73:211-8. doi:10.1016/j.neurobiolaging.2018.09.027

[49] Li JN, Liu XL, Li L. Prefrontal GABA and glutamate levels correlate with impulsivity and cognitive function of prescription opioid addicts: A (1) H-magnetic resonance spectroscopy study. Psychiatry Clin Neurosci 2020;74(1):77-83. doi:10.1111/pcn.12940 
491

492

493

494

495

496
[50] Chen Y, Gao X, Liu Q, Zeng L, Zhang K, Mu K, et al. Alpha-asarone improves cognitive function of aged rats by alleviating neuronal excitotoxicity via GABAA receptors. Neuropharmacology 2020;162:107843. doi:10.1016/j.neuropharm.2019.107843 
Figures
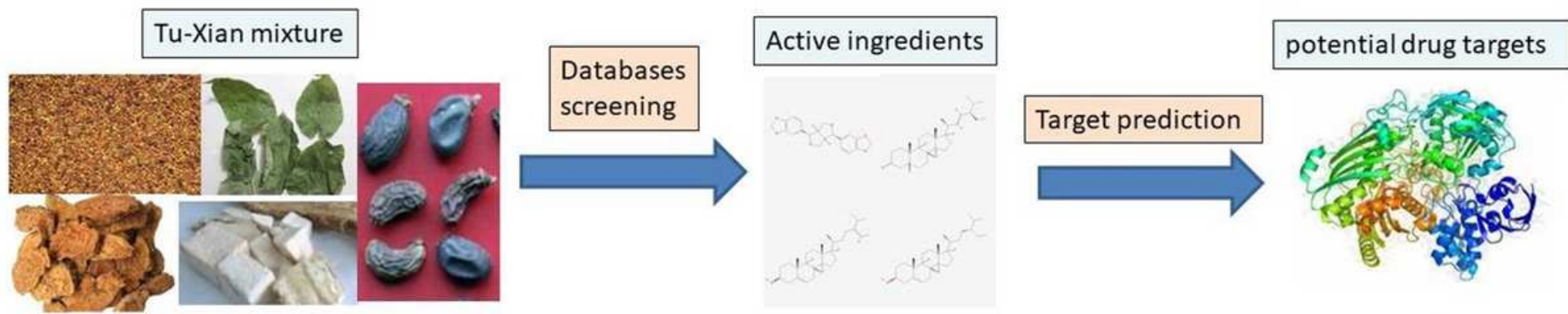

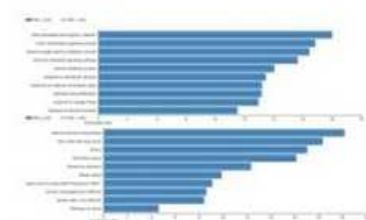

Mechanism of action
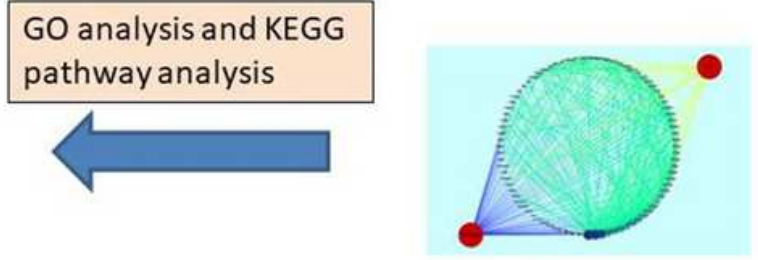

Network analysis

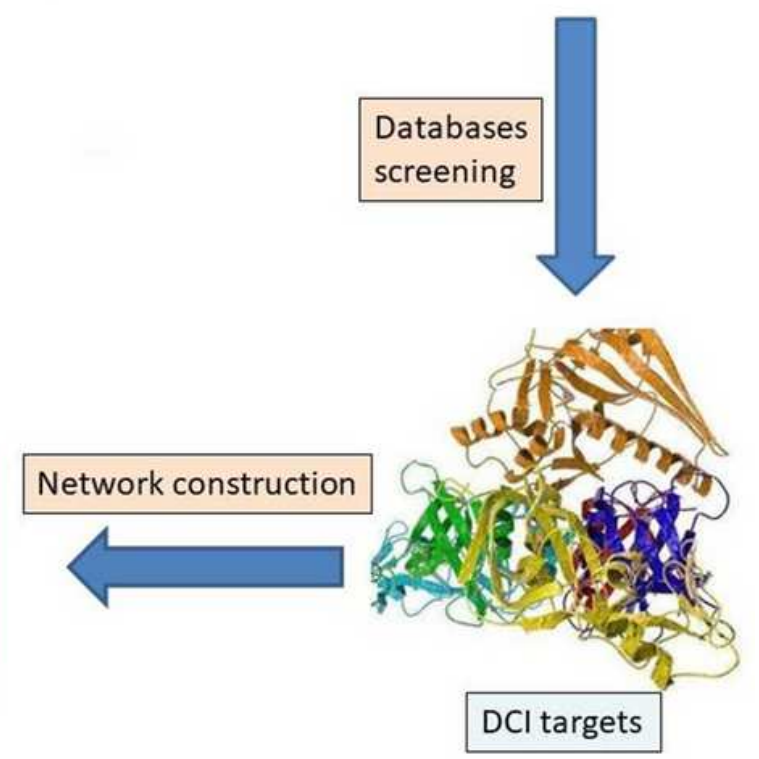

Figure 1

Workflow of the approach 


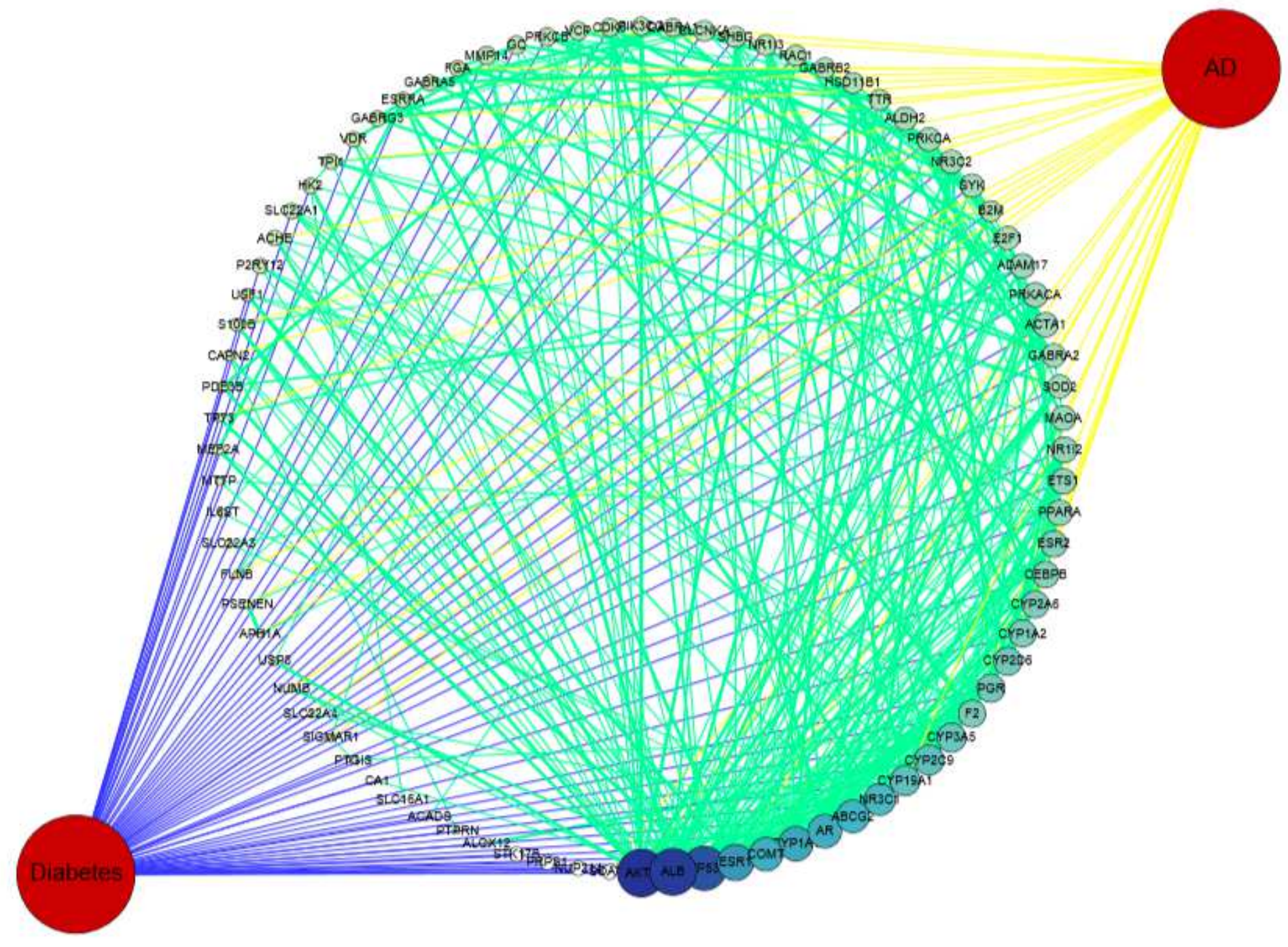

Figure 2

The PPI between potential therapeutic targets of diabetes and AD 
DNA-templated transcription, initiation

multi-multicellular organism process

reactive oxygen species metabolic process

hormone-mediated signaling pathway

steroid metabolic process

response to xenobiotic stimulus

response to molecule of bacterial origin

epithelial cell proliferation

response to oxygen levels

response to steroid hormone

Enrichment ratio

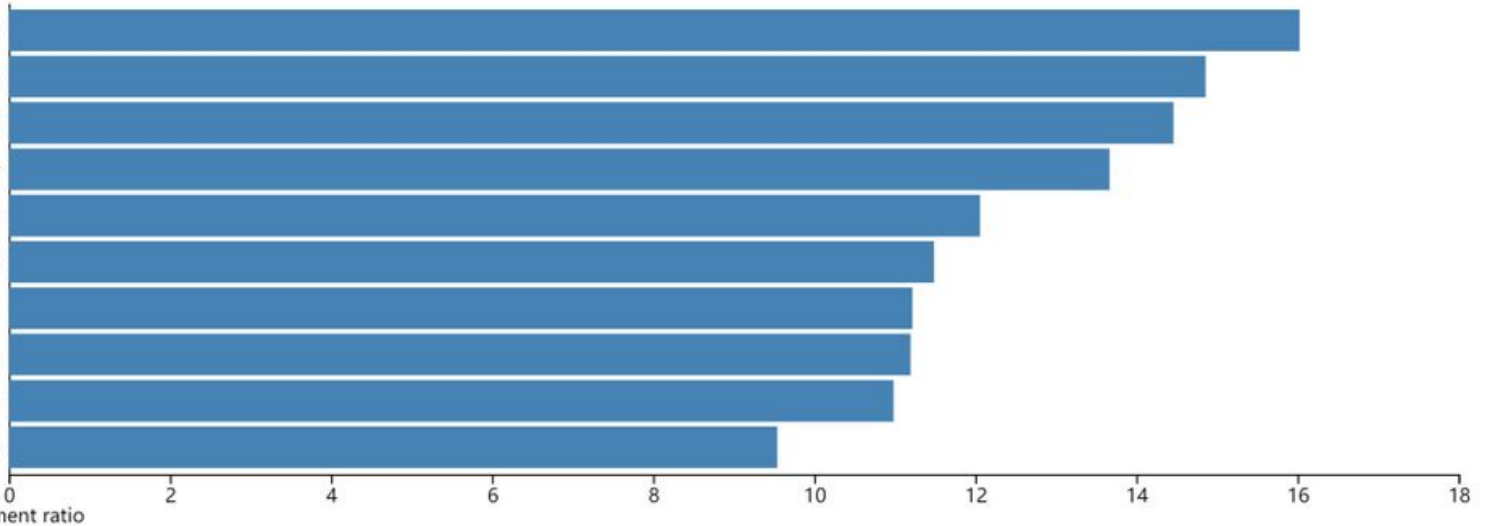

FDR $\leq 0.05$

FDR $>0.05$

Steroid hormone biosynthesis

Non-small cell lung cancer

Glioma

Pancreatic cancer

Endocrine resistance

Breast cancer

aposi sarcoma-associated herpesvirus infect...

Human cytomegalovirus infection

Epstein-Barr virus infection

Pathways in cancer

$\stackrel{0}{\text { Enrichment ratio }}$

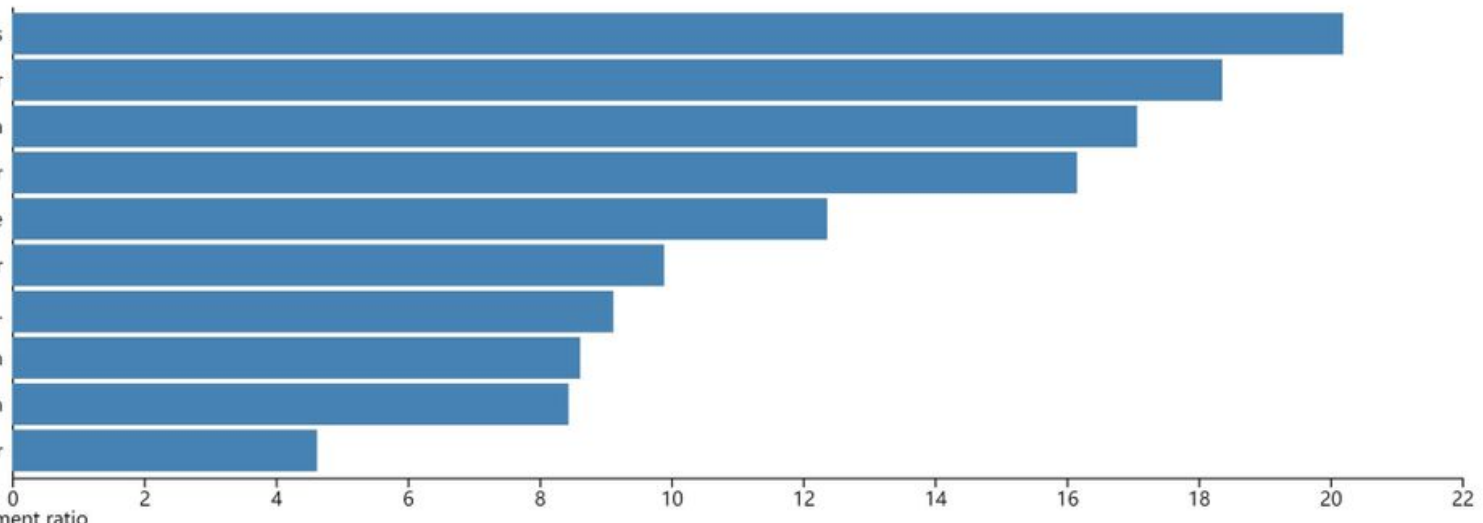

\section{Figure 3}

GO enrichment analysis and KEGG pathway analysis of diabetes key targets 
amma-aminobutyric acid signaling pathway synaptic transmission, GABAergic

drug catabolic process

inorganic anion transport

hormone metabolic process

anion transmembrane transport

regulation of membrane potential

steroid metabolic process

regulation of neurotransmitter levels

neuron death

Enrichment ratio

20

$40 \quad 60$

$80 \quad 100$

120

140

160

FDR $\leq 0.05 \quad$ FDR $>0.05$

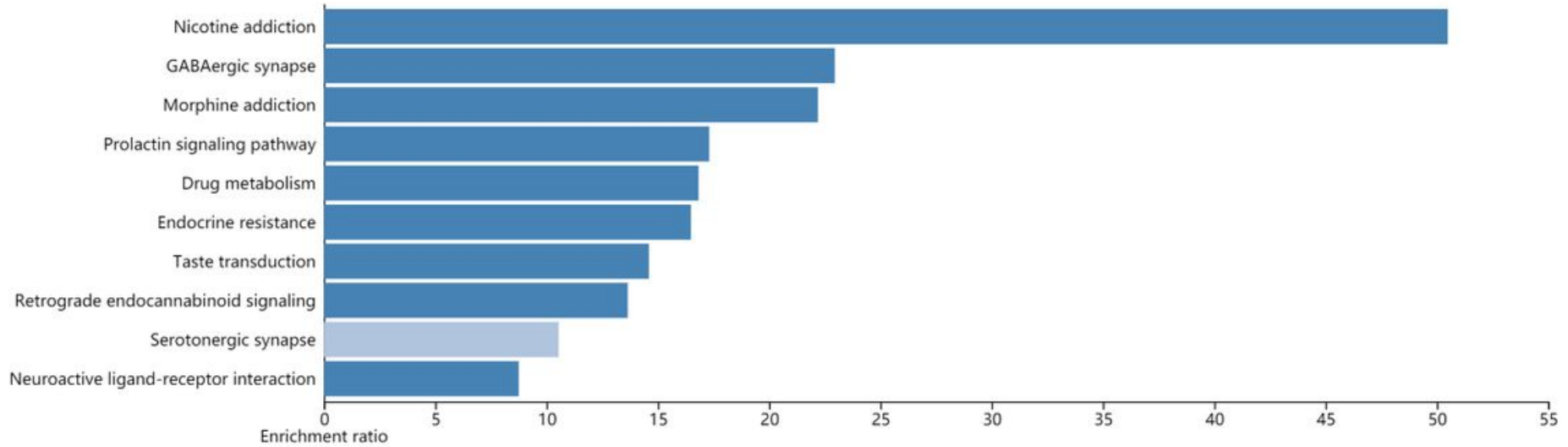

\section{Figure 4}

GO enrichment analysis and KEGG pathway analysis of AD key targets

\section{Supplementary Files}

This is a list of supplementary files associated with this preprint. Click to download.

- supplementary.rar 